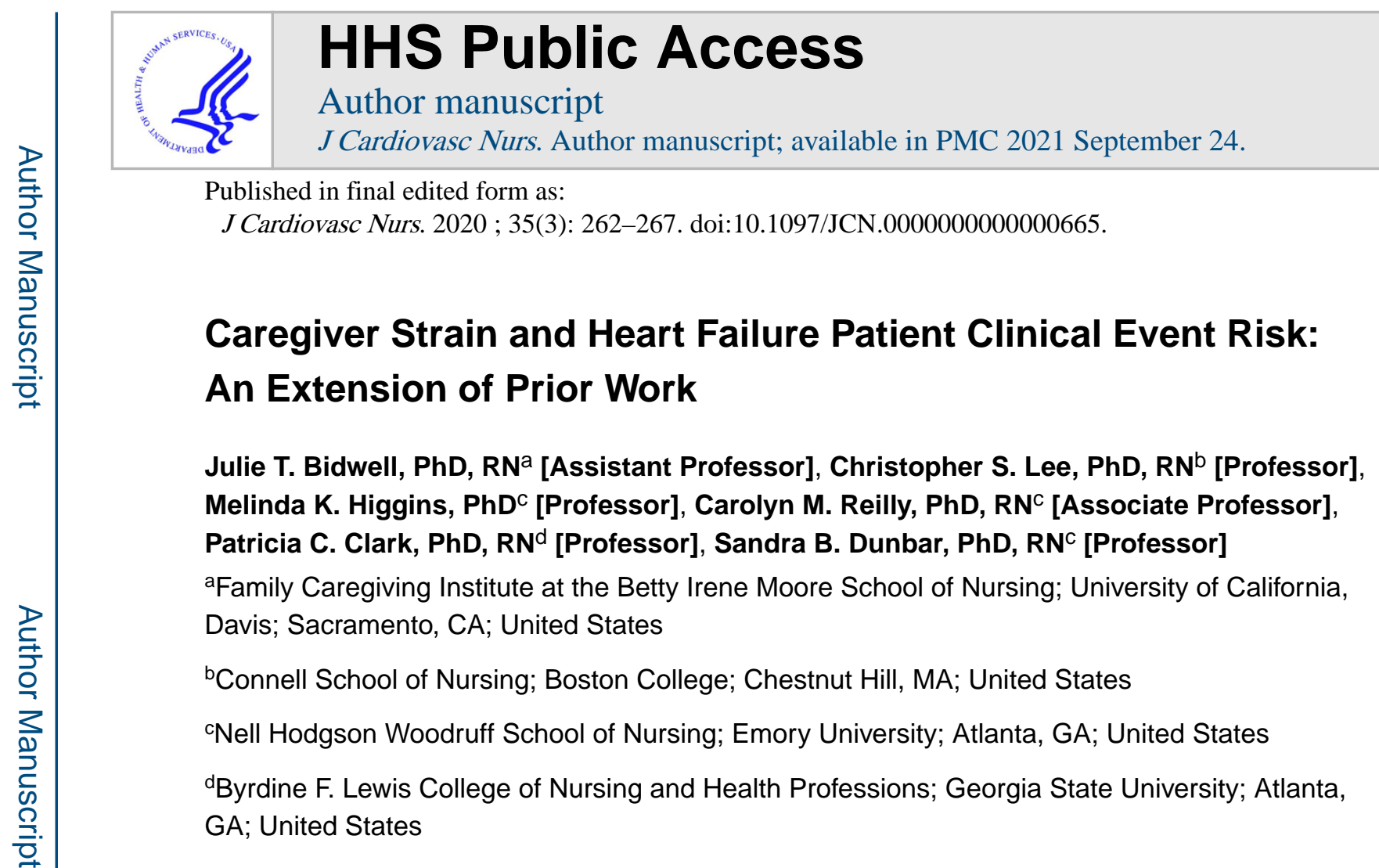

\title{
Abstract
}

Background: In a study of Italian heart failure (HF) patient-caregiver dyads, greater caregiver strain significantly predicted lower patient clinical event-risk.

Objective: The purpose of this secondary analysis was to examine this relationship in a sample from the United States.

\begin{abstract}
Methods: Data came from 92 dyads who participated in a self-care intervention. Logistic regression was used to test the relationship between baseline strain (Bakas Caregiving Outcomes Scale, divided into tertiles) and patient likelihood of events (HF hospitalization/emergency visit or all-cause mortality) over 8 months.
\end{abstract}

Results: Nearly half ( $n=40,43.5 \%$ ) of patients had an event. High (versus low) caregiver strain was associated with a $92.7 \%$ event-risk reduction, but with substantial variability around the effect (OR 0.07, 95\% CI 0.01-0.63, $\mathrm{p}=0.02$ ).

Conclusions: Although findings were similar to the Italian study, the high degree of variability and contrasting findings to other studies signal a level of complexity that warrants further investigation.

\section{MeSH Terms/Keywords:}

Heart Failure; Hospitalization; Mortality; Caregivers; Caregiver Strain/Burden

Corresponding Author: Julie T. Bidwell, PhD, RN; $245048^{\text {th }}$ Street \#2637, Sacramento, CA 95817; phone: 1+916-703-3706; fax: 1+916-734-4116; jtbidwell@ucdavis.edu. 


\section{INTRODUCTION}

Even with substantial research and health policy efforts, the burden of serious HF-related clinical events (e.g. hospitalization, death) remains high. ${ }^{1}$ Although many persons with HF depend on family caregivers to help them with HF self-care behaviors that are critical to maintaining clinical stability, ${ }^{2}$ few HF risk models include caregiver-level factors. ${ }^{3}$ Caregiver strain (i.e. caregiver burden, defined as subjectively-reported physical, psychological, social, and/or financial distress experienced as a result of caregiving ${ }^{4}$ ) is of particular interest, given its prevalence and association with increased morbidity and mortality for caregivers, and potential for reduced ability to provide appropriate support to the person with $\mathrm{HF}^{5,6}$ In a previous systematic review of caregiver well-being and HF patient outcomes, few studies examined the relationship between caregiver strain and patient clinical events. However, a consistent positive relationship was identified (lower strain associated with fewer events and vice versa). ${ }^{7}$ In contrast, when this relationship was examined in a large prospective Italian study of HF patient-caregiver dyads, the opposite relationship was found. ${ }^{8}$ The purpose of this analysis was to examine the relationship between caregiver strain and HF patient clinical events in a sample of HF dyads from the United States.

\section{METHODS}

\section{Study Design}

Data for this secondary analysis were from the Education and Support Interventions to Improve Self-Care clinical trial (ENSPIRE; ClinicalTrials.gov identifier: NCT00166049). ENSPIRE was a 3-group randomized controlled trial comparing patient-family education or patient-family education plus autonomy supportive communication to usual care in improving patient HF self-care behaviors. The study design and primary results have been published previously. ${ }^{9}$ In short, persons with HF and their family caregivers were recruited in dyads from 3 outpatient HF clinics in the Southern United States. Persons with HF were included if they were aged 30-79, New York Heart Association Class II-III, and on optimal medical therapy. Family caregivers were included in they were aged $\geq 19$, the primary family member or friend assisting with HF management, and had $\geq 2$ caregiving interactions weekly. Data were collected at baseline and at 4 and 8 months. The study protocol was approved by the Institutional Review Boards at each site, and all participants provided written informed consent.

\section{Measurement}

Patient and caregiver demographic information was collected via self-report; patient clinical characteristics were abstracted from the medical record. The primary composite outcome for this analysis was the self-reported occurrence of any event during follow-up. Events were defined as hospitalization for HF, emergency department (ED) visit for HF, or all-cause death, collected at 4 and 8 months and verified against medical record data at the index site. The primary independent variable for this analysis was baseline caregiver strain as measured by the 15 -item Bakas Caregiving Outcomes Scale $(\mathrm{BCOS})^{10}$ divided by tertiles based on 
observed distribution characteristics into low, moderate, and high strain. The Cronbach's alpha for the BCOS in this sample was 0.94 .

\section{Statistical Analysis}

Because sample size precluded a comprehensive model controlling for all known confounders of HF event-risk, we used randomization of the study groups and a group-bystrain interaction design to indirectly control for confounding while maintaining model parsimony. In the original study, there were no significant demographic or clinical differences by intervention group. ${ }^{9}$ However, to confirm that randomization was also successful in dyads with clinical events data, and to identify any additional caregivingrelated characteristics that may be different across groups and would need to be controlled in the final model, basic comparisons were used to test for significant differences across the three intervention study groups (oneway ANOVA or non-parametric Kruskal-Wallis H-test for continuous variables, chi-square test for categorical variables). Logistic regression was used to quantify the relationship between baseline caregiver strain (high or moderate versus low) and likelihood of any patient clinical event during 8 months follow-up, controlling for intervention group, group-by-strain interaction, and recent hospitalization for HF (within 4 months prior to enrollment), given that prior hospitalization is one of the most consistent predictors of future events in HF. ${ }^{11,12}$

\section{RESULTS}

\section{Sample characteristics}

Sociodemographic characteristics.-Ninety-two patient-caregiver dyads from the original study had data pertaining to clinical events, and were included in this secondary analysis. Patient and caregiver factors were well-randomized across the study groups (Supplemental Table). The sample demographics are displayed in the Table. In short, persons with $\mathrm{HF}$ and caregivers were in their mid-fifties, majority African American, and well-educated. Most persons with HF were male, most caregivers were female, and half of the dyads were spousal or partnered. The vast majority of patients had non-ischemic HF and were NYHA Class II at the time of enrollment, and $40 \%$ entered the study having been hospitalized in the previous 4 months for HF.

Patient Clinical events.-Over the course of study follow-up, just over half of persons with HF (56.5\%, n=52) had no qualifying events (HF hospitalization or ED visit, or allcause death). Of those who did have one or more qualifying events during follow-up (43.5\%, $\mathrm{n}=40$ ), the most common event was HF hospitalization. Cumulatively, there were $71 \mathrm{HF}$ hospitalizations, $57 \mathrm{HF}$ ED visits not resulting in hospitalization, and 6 deaths reported during the study period.

Caregiver strain.-The average BCOS score for caregivers was 65.2 \pm 15.7 (median 60, sample range $31-105$, possible range 15-105, higher scores indicate lower strain). The distribution of strain contained a high concentration of participants at the median score of $60(20.0 \%, \mathrm{n}=18)$, bracketed by well-distributed tails, supporting the use of tertiles to characterize the sample. The average and range for the low, moderate, and high strain tertiles 


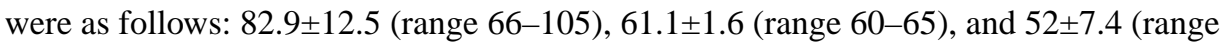
31-59), respectively.

Relationship between Caregiver Strain and Patient Clinical Events.-The multivariate logistic regression model testing the relationship between baseline caregiver strain and likelihood of the person with HF experiencing HF hospitalization, HF ED visit, or all-cause death during the 8 month follow-up period had acceptable fit (Model $\chi^{2}=$ 19.6, $\mathrm{p}=0.02$; $\mathrm{p}$-values for Pearson and Hosmer-Lemeshow goodness-of-fit tests both 0.99). There was no significant difference in patient event-risk by intervention group. There was no significant difference in likelihood of clinical events for patients with caregivers who reported moderate strain, as compared to patients with caregivers who reported low strain (OR 0.29, 95\% CI 0.04-2.43, p = 0.26, effect size [Cohen's d] = 0.67). Patients with caregivers who reported high strain (as compared to low strain) were significantly less likely to experience a clinical event, although the variability around the effect was substantial (OR $0.07,95 \%$ CI 0.01-0.63, p = 0.02; effect size [Cohen's d] = 1.44; Figure) .

\section{DISCUSSION}

In this study of diverse HF patient-caregiver dyads from the Southern United States, we tested the relationship between caregiver strain and patient risk for clinical events (ED or hospital visit for HF, or all-cause death), and found a significant inverse (but widely variable) relationship between caregiver strain and patient clinical events (i.e. higher strain related to lower risk of patient events and vice versa) over 8 months follow-up. Although similar to our recent study in Italian HF dyads, ${ }^{8}$ this finding is surprising given its apparent discordance with other studies in HF that have demonstrated a positive relationship between care strain and patient events. ${ }^{7}$ This presents two important questions: (1) what mechanisms might explain the inverse relationship between caregiver strain and patient event-risk in our two studies and (2) what might explain variability in this effect, both within this study and across studies in HF.

There are a number of phenomena from the broader caregiving literature that may explain the inverse relationship we observed. Most intuitively, increased care strain may be reflective of higher intensity of caregiving activities that are known to be burdensome for caregivers, but also beneficial to the health of the person with chronic disease. ${ }^{5}$ If this is the case, reaping these benefits is concerning for the long-term health of the caregiver, and likely unsustainable for patient outcomes over the trajectory of disease. ${ }^{5}$ Care strain can also occur when caregivers have reached the limits of what they can provide the patient, and yet the patient still reports unmet care needs, which may eventually lead to worsening patient outcomes. ${ }^{13}$ Reaching this point in the caregiving/HF trajectory may partly explain the opposing relationship previously reported in other HF studies (i.e. higher care strain associated with greater event-risk). ${ }^{7}$ Since patients in this study were less likely to have a clinical event when their caregiver reported higher strain, it is possible that this sample of caregivers had not been exposed to high-strain caregiving for a long period of time.

Considering the other direction of the inverse relationship, lower care strain predicting higher patient events may be a function of caregivers stepping back from intense caregiving 
activities that might otherwise provide tangible benefit to the person with HF. For example, caregivers may be pressed for time or overwhelmed with other responsibilities and less able to engage in intense caregiving. Or they may choose to limit potentially beneficial engagement in HF management in an effort to avoid infringing on the autonomy of the person with HF (e.g. concerns of being perceived as critical or controlling) or to protect the existing dynamics of their relationship. ${ }^{14,15}$ It is also possible that some persons with HF who would benefit from family member assistance attempt to conceal their needs or the severity of their symptoms from their family member in an effort not to burden them. Concealment can contribute to poor health-related communication and reduced dyadic agreement on patient symptoms - both of which may hamper optimal self-care and lead to poor outcomes. ${ }^{16,17}$

Given the wide confidence intervals in this study and contrasting findings in other studies, examining factors that may explain variability in the effect is an important direction for future research. Caregiver strain is highly heterogeneous, both in terms of how it is conceptualized and measured, and in terms of the numerous factors - independent or in combination - that contribute to its development in caregivers. ${ }^{5} \mathrm{HF}$ event-risk is also heterogeneous, with numerous disease-specific and individual, relational, and contextual factors driving outcomes. ${ }^{3}$ Thus, wide variability in the effect is likely attributable to multiple factors, and the most productive next steps for research might be to move away from quantifying average effects, and begin a targeted examination of specific factors (e.g. patient, caregiver, relational, social/contextual, cultural, healthcare system characteristics/ access, time/trajectory) that explain variability in the effect.

When considering overall implications of our findings, it is perhaps most critical to emphasize that caregiver strain, regardless of any potential downstream benefits for patient health, is quantifiably hazardous for the caregiver. In addition to compromised physical and psychological health status, there is evidence that the experience of caregiving particularly higher-strain caregiving - is associated with increased cardiovascular risk and mortality. ${ }^{5,18,19}$ Long-term and/or intense caregiving for a spouse/partner predicts increased incidence of cardiovascular disease in adults that were previously healthy, ${ }^{19}$ and those who report caregiving strain have higher risk for mortality than noncaregivers. ${ }^{20}$ As cardiovascular clinicians and researchers, we should never be comfortable with calculus that involves exposing one population to increased cardiovascular disease risk and mortality (caregivers) in our efforts to improve the clinical outcomes for another population (persons with HF). To frame it in the context of US cardiovascular health goals, we make questionable progress if we move towards our HF target of reducing rehospitalization (Heart Disease and Stroke Objective 24) while losing critical ground on increasing cardiovascular health in the overall US population (Heart Disease and Stroke Objective 1). ${ }^{21}$ In order to support more equitable health outcomes in the context of our growing population of family caregivers, theoretical frameworks and family-centered care interventions that seek to benefit or balance the health of both members of the care dyad may be particularly valuable in guiding research and clinical care. ${ }^{17,22}$

This study does have limitations, most notably the small sample size, which precludes independently controlling for all known predictors of HF event-risk. Although we did use 
randomization of the groups and a group-by-strain interaction design to indirectly control for confounders and intervention effects, the lack of robust controls is a serious limitation and results must be interpreted with caution. Larger observational studies are needed to confirm the existence/directionality of this relationship and, perhaps more importantly, explain the variability that surrounds it. The use of self-reported clinical outcomes should also be considered a limitation, although self-reported healthcare utilization data is widely used in epidemiological studies, with acceptable levels of agreement between self-report and medical records data (exceeding $90 \%$ for hospitalization). ${ }^{23}$ Similarly, the original study did involve verification of events with clinical chart reviews (for those re-hospitalized at the index institution only), with high agreement with self-report.

There are also notable limitations associated with the demographics of the study sample that must be considered. First, NYHA Class IV patients were excluded, which means that these results cannot be generalized to caregiving for advanced HF, which is more likely to be of greater intensity and involve more frequent clinical events. Second, most caregivers in this study were women, and there is evidence that the HF caregiving experience differs by gender, with women experiencing greater distress. ${ }^{24}$ While the Italian study had a greater proportion of male caregivers $(33 \%),{ }^{8}$ that sample was also largely female; thus, an examination of gender differences in care strain and patient event-risk in a larger, balanced sample is warranted. Third, the patients and caregivers in this sample were relatively young, and the caregivers were relatively healthy (i.e. had few comorbidities). Given that multimorbidity is an increasing challenge for both HF patients and their caregivers, particularly in the context of aging, one important direction for advancing this work may be to examine potential compounding effects of multiple chronic conditions and declining caregiver health on the evolution of care strain and patient event-risk.

The measurement of caregiver strain and the division of the strain variable into tertiles for analysis may also be considered limitations. Although the BCOS is frequently used in $\mathrm{HF}$ as a measure of caregiver strain/burden and overlaps conceptually with a foundational definition of caregiver strain/burden from the broader caregiving literature, ${ }^{4}$ its architects do not explicitly describe it as a measure of care strain, nor is it commonly used outside of HF/ stroke. However, systematic reviews of caregiver strain/burden instruments do include the BCOS as a measure of caregiver burden, with good evidence for reliability and validity. ${ }^{25}$ Despite this, it should be noted that the Italian analysis used a different instrument to measure strain, which tempers the strength of conclusions that can be drawn collectively across the two studies. The Italian analysis also did not categorize the strain variable for analysis, which should also be considered a limitation. The use of quantiles to examine effects that occur at particularly high or low levels of a variable is a common approach when statistical power is a concern, and more pronounced effects are expected at the "ends" of a distribution. ${ }^{26}$ Both were the case in this analysis, as sample size was considerably smaller than the Italian study, and adverse outcomes are typically more pronounced in high-intensity, high-strain caregiving contexts.

Despite these limitations, the major strength of this study is that it is an extension of previous work, and the effects we observed in this study using a diverse sample of HF dyads from the United States are similar to the effects we observed in our prior study using 
an Italian sample. As such, this work builds upon an existing line of inquiry and provides rationale and direction for continued study.

\section{CONCLUSIONS}

In this diverse sample of HF patient-caregiver dyads from the United States, we found that caregiver strain and risk for patient clinical events were inversely related (higher care strain was associated with lower risk for events and vice versa). These findings are similar to that of our previous study conducted in Italian dyads. ${ }^{8}$ However, wide variability in the effect, coupled with contrasting effects found in other studies of persons with $\mathrm{HF}$ and caregivers, signal a level of complexity that warrants further study. In particular, research is needed to examine variability around this relationship, and, most importantly, identify which modifiable factors might be targeted to support the best-case scenario in care dyads caregivers with little/no strain and patients with long-term event-free survival. Theories and care models that include both the patient and the caregiver are likely best-equipped to guide research to uncover these important protective mechanisms. ${ }^{17}$

\section{Supplementary Material}

Refer to Web version on PubMed Central for supplementary material.

\section{Conflicts of Interest and Source of Funding:}

The authors declare no conflict of interest. This work was supported by the National Institutes of Health/ National Institute of Nursing Research T32NR012715 (Bidwell) and R01R08800 (Dunbar); the Public Health Service M01 RR0039 (Dunbar); and the National Institutes of Health/National Center for Research Resources UL1 RR025008 (Dunbar). The remaining authors declare no sources of funding. The content is solely the responsibility of the authors and does not necessarily represent the official views of the funders.

\section{References}

1. Benjamin EJ, Muntner P, Alonso A, et al.Heart disease and stroke statistics-2019 update: A report from the American Heart Association. Circulation. 2019:CIR0000000000000659.

2. Buck HG, Harkness K, Wion R, et al.Caregivers' contributions to heart failure self-care: a systematic review. Eur J Cardiovasc Nurs. 2015;14(1):79-89. [PubMed: 24399843]

3. Rahimi K, Bennett D, Conrad N, et al.Risk prediction in patients with heart failure: A systematic review and analysis. JACC Heart Fail. 2014;2(5):440-446. [PubMed: 25194291]

4. Zarit SH, Todd PA, Zarit JM. Subjective burden of husbands and wives as caregivers: a longitudinal study. Gerontologist. 1986;26(3):260-266. [PubMed: 3721233]

5. Schulz R, Beach SR, Czaja SJ, Martire LM, Monin JK. Family caregiving for older adults. Annu Rev Psychol. [in press].

6. Dionne-Odom JN, Hooker SA, Bekelman D, et al.Family caregiving for persons with heart failure at the intersection of heart failure and palliative care: A state-of-the-science review. Heart Fail Rev. 2017(2017204):1-15. [PubMed: 27844178]

7. Bidwell JT, Lyons KS, Lee CS. Caregiver well-being and patient outcomes in heart failure: A meta-analysis. J Cardiovasc Nurs. 2017;32(4):372-382. [PubMed: 27617564]

8. Bidwell JT, Vellone E, Lyons KS, et al.Caregiver determinants of patient clinical event risk in heart failure. Eur J Cardiovasc Nurs. 2017:1474515117711305.

9. Dunbar SB, Clark PC, Reilly CM, et al.A trial of family partnership and education interventions in heart failure. J Card Fail. 2013;19(12):829-841. [PubMed: 24331203] 
10. Bakas T, Champion V, Perkins SM, Farran CJ, Williams LS. Psychometric testing of the revised 15-item Bakas Caregiving Outcomes Scale. Nurs Res. 2006;55(5):346-355. [PubMed: 16980835]

11. Dharmarajan K, Hsieh AF, Kulkarni VT, et al.Trajectories of risk after hospitalization for heart failure, acute myocardial infarction, or pneumonia: Retrospective cohort study. BMJ. 2015;350:h411. [PubMed: 25656852]

12. Jencks SF, Williams MV, Coleman EA. Rehospitalizations among patients in the Medicare fee-forservice program. N Engl J Med. 2009;360(14):1418-1428. [PubMed: 19339721]

13. Nieuwenhuis AV, Beach SR, Schulz R. Care recipient concerns about being a burden and unmet needs for care. Innov Aging. 2018;2(3):1-10. [PubMed: 30480150]

14. Horowitz A, Silverstone BM, Reinhardt JP. A conceptual and empirical exploration of personal autonomy issues within family caregiving relationships. Gerontologist. 1991;31(1):2331. [PubMed: 2007472]

15. Dunbar SB, Clark PC, Quinn C, Gary RA, Kaslow NJ. Family influences on heart failure self-care and outcomes. J Cardiovasc Nurs. 2008;23(3):258-265. [PubMed: 18437068]

16. Miller LM, Lyons KS, Bennett JA. Incongruent perceptions of pain and physical function among families living with lung cancer. Support Care Cancer. 2015;23(9):2755-2762. [PubMed: 25669967]

17. Lyons KS, Lee CS. The Theory of Dyadic Illness Management. J Fam Nurs. 2018:1074840717745669.

18. Grant JS, Graven LJ. Problems experienced by informal caregivers of individuals with heart failure: An integrative review. Int J Nurs Stud. 2018;80:41-66. [PubMed: 29353711]

19. Capistrant BD, Moon JR, Berkman LF, Glymour MM. Current and long-term spousal caregiving and onset of cardiovascular disease. J Epidemiol Community Health. 2012;66(10):951-956. [PubMed: 22080816]

20. Schulz R, Beach SR. Caregiving as a risk factor for mortality: the Caregiver Health Effects Study. JAMA. 1999;282(23):2215-2219. [PubMed: 10605972]

21. Pahigiannis K, Thompson-Paul AM, Barfield W, et al.Progress toward improved cardiovascular health in the United States. Circulation. 2019;139(16):1957-1973. [PubMed: 30986104]

22. Park M, Giap TT, Lee M, Jeong H, Jeong M, Go Y. Patient- and family-centered care interventions for improving the quality of health care: A review of systematic reviews. Int J Nurs Stud. 2018;87:69-83. [PubMed: 30056169]

23. Roberts RO, Bergstralh EJ, Schmidt L, Jacobsen SJ. Comparison of self-reported and medical record health care utilization measures. J Clin Epidemiol. 1996;49(9):989-995. [PubMed: 8780606]

24. Rohrbaugh MJ, Cranford JA, Shoham V, Nicklas JM, Sonnega JS, Coyne JC. Couples coping with congestive heart failure: role and gender differences in psychological distress. J Fam Psychol. 2002;16(1):3-13. [PubMed: 11915408]

25. Deeken JF, Taylor KL, Mangan P, Yabroff KR, Ingham JM. Care for the caregivers: a review of self-report instruments developed to measure the burden, needs, and quality of life of informal caregivers. J Pain Symptom Manage. 2003;26(4):922-953. [PubMed: 14527761]

26. Preacher KJ, Rucker DD, MacCallum RC, Nicewander WA. Use of the extreme groups approach: a critical reexamination and new recommendations. Psychol Methods. 2005;10(2):178-192. [PubMed: 15998176]

27. Jann BPlotting regression coefficients and other estimates. The Stata Journal. 2014;14(4):708-737. 


\section{What's New?}

- In this study, higher caregiver strain (burden) predicted lower HF patient clinical events (hospitalization for HF, emergency department visit for HF, or all-cause death).

- $\quad$ This secondary analysis, conducted in a sample of HF patient-caregiver dyads from the United States, corroborates findings from a prior study of Italian HF dyads.

- $\quad$ Research guided by theories/models designed to balance patient and caregiver outcomes may be most useful in examining the substantial variability around this relationship and uncovering mechanisms that promote the health of both dyad members. 


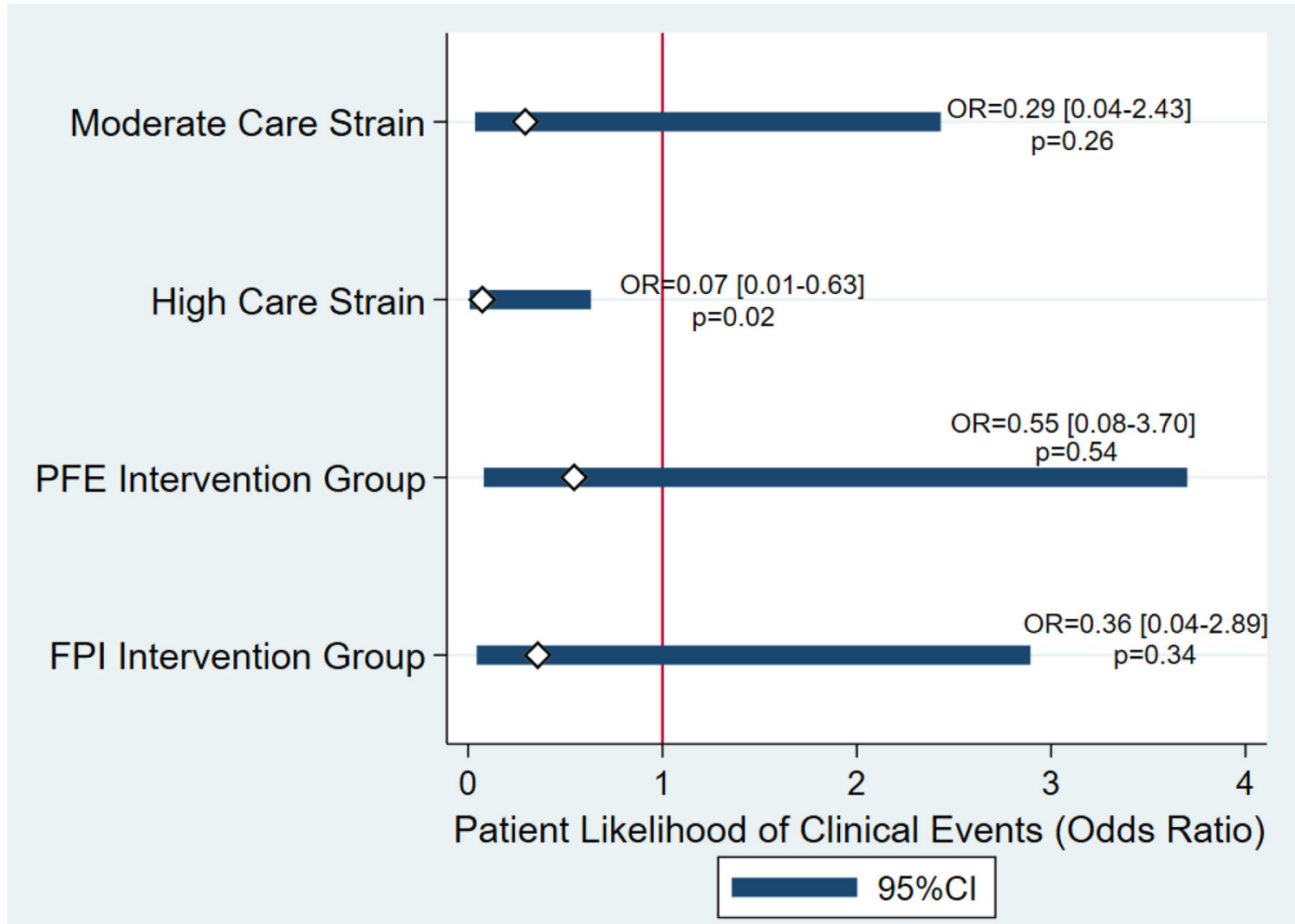

Odds ratios and $95 \%$ confidence intervals for the effects of baseline caregiver strain (moderate and high strain tertiles as opposed to low strain) and intervention grouping (Patient-Family Education [PFE] and Family Partnership Intervention [FPI] as opposed to usual care) on patient risk for any clinical event (HF hospitalization, HF emergency visit, or all-cause mortality) over 8 months follow-up. Data visualized in StataMPv15 using coefplot. ${ }^{27}$ 
Table:

Characteristics of the Sample ( $\mathrm{n}=92$ dyads)

\begin{tabular}{|l|l|l|}
\hline & Patient mean \pm SD or n(\%) & Caregiver mean \pm SD or $\mathbf{n}(\%)$ \\
\hline Age & $56.3 \pm 10.1$ & $53.4 \pm 12.4$ \\
\hline Female Gender & $35(38.0)$ & $75(82.4)$ \\
\hline African American Race & $58(63.0)$ & $59(64.8)$ \\
\hline College Education or Higher & $45(48.9)$ & $44(48.4)$ \\
\hline Spousal Caregiver & & $47(51.7)$ \\
\hline Charlson Comorbidity Score & $3.2 \pm 2.2$ & $1.0 \pm 1.5$ \\
\hline HF Duration 25 Years & $47(51.1)$ & \\
\hline Ejection Fraction (\%) & $27.2 \pm 14.4$ & \\
\hline NYHA Class III (versus II) & $24(26.1)$ & \\
\hline Previous HF Hospitalization & $35(39.8)$ & \\
\hline Caregiver Strain (BCOS) & $65.2 \pm 15.7$ & \\
\hline
\end{tabular}

Hospitalization for heart failure within 4 months prior to study enrollment

Key: $\mathrm{BCOS}=$ Bakas Caregiving Outcomes Scale; $\mathrm{HF}=$ heart failure; NYHA Class $=$ New York Heart Association Functional Class. 